\title{
Stochastic theory of quantum vortex on a sphere
}

\author{
Hiroshi Kuratsuji \\ Research Organization of Science and Engineering, \\ Ritsumeikan University-BKC, Kusatsu City, 525-8577, Japan
}

(Dated: October 10, 2018)

\begin{abstract}
A stochastic theory is presented for a quantum vortex that is expected to occur in superfluids coated on two dimensional sphere $\mathrm{S}^{2}$. The starting point is the canonical equation of motion ( the Kirchhoff equation) for a point vortex, which is derived using the time-dependent LandauGinzburg theory. The vortex equation, which is equivalent to the spin equation, turns out to be the Langevin equation, from which the Fokker-Planck equation is obtained by using the functional integral technique. The Fokker-Planck equation is solved for several typical cases of the vortex motion by noting the specific form of pinning potential. An extension to the non-spherical vortices is briefly discussed for the case of the vortex on plane and pseudo-sphere.

PACS numbers: 05.40.-a, 67.25.dk, 67.25.dr
\end{abstract}

\section{INTRODUCTION}

The study of vortex dynamics has been one of central subjects in classical physics[1]. The modern interest of vortex phenomena is motivated by the quantum fluids, namely, the quantum vortex in various contexts, which are considered to be defects inherent in the complex order parameters describing the quantum fluids. Specifically, the dynamics of point vortex has been extensively investigated so far [2-4]. The dynamics of vortex string has been also studied [5]. Besides the conventional vortex in superfluid He4, the more complicated textural structure in He3 has been explored ([6]). Furthermore even the vortex in the cosmological scale has been explored(e.g. [7]) .

In conventional treatment the vortex is an object defined on Euclidean space of 2 and/or 3 dimension. The vortex defined on curved space rather than flat Euclidean space is possible to occur. Indeed, the classical counterpart of non-planer vortex has been given in several contexts starting with the purely classical hydrodynamical procedure,see e.g. [8, 9].

On the other hand, it is known that in condensed matter physics the vortex motion is influenced by randomness caused by e.g. the temperature fluctuation and the presence of the interaction with the various sorts of impurities. For such cases, we need to treat it within a framework of stochastic theory. Indeed, the stochastic theory of vortex has been investigated in connection with the superconductivity [10] .

The purpose of this note is to present a general formalism for stochastic theory of quantum vortex that is constrained on the two dimensional manifold; specifically, two dimensional sphere $\mathrm{S}^{2}$. The essential point is to follow an analogy between vortex and ferromagnetic spin: that is realized for a single magnetic "domain" [11]. In order to achieve this, we first derive the equation of motion for the vortex center on these manifold; the canonical (Kirchhoff) equation by using the time-dependent Landau-Ginzburg theory [12], that is described by the complex order parameter on $\mathrm{S}^{2}$ (Section 2 ) . Then the equation of motion for the vortex is con- verted to the Langevin equation by noting the analogy between the spherical vortex and the spin in the presence of the random force (Section 3). From this analogy, the Fokker-Planck equation is derived by adopting the functional integral technique. The functional integral used here is borrowed from the procedure that was developed for the statistical theory of wave propagation in random media 13, 14]. In section 4, we show the solutions of the FP equation for a typical form of pinning potential. In the section 5, we give a brief sketch of an extension of the spherical vortex to its variants; the planer vortex and then pseudo-sphere vortex.

\section{EQUATION OF MOTION FOR QUANTUM VORTEX}

We start with the time-dependent Landau-Ginzburg Lagrangian, that is expressed in terms of the order parameter $\psi$ :

$$
L=\int\left[\frac{i \hbar}{2}\left(\psi^{*} \frac{\partial \psi}{\partial t}-\text { c.c. }\right)-\mathcal{H}\left(\psi \cdot \psi^{*}\right)\right] d \sigma .
$$

This form is the same as that was used for the superfluid which is defined on a flat plane 12$]$. Here $\psi(\mathbf{r}, t)$ is defined on 2-sphere $S^{2}$ with radius $|\mathbf{r}|=a$ and the integral $\int d \sigma$ is taken over $S^{2}$, the coordinate of which is denoted by $\mathbf{r}$ written in terms of the Cartesian form. $\mathcal{H}$ represents the Hamiltonian density which consists of the kinetic and potential energy;

$$
\mathcal{H}=\frac{\hbar^{2}}{2 m} \nabla \psi^{*} \nabla \psi+V\left(\psi^{*}, \psi\right)
$$

where $\nabla$ means the derivative with respect to the spherical coordinate and $m$ means the mass of constituent particles (e.g. He4 atoms).

Let us consider the case that a single vortex is created, for which the order parameter is written by a form such that

$$
\psi(\mathbf{r}, t)=\psi(\mathbf{r}-\mathbf{R}(t)) .
$$


Here $\mathbf{R}$ denotes the time-dependent vortex center satisfying $|\mathbf{R}|=a$. With this parametric form we have

$$
\frac{i \hbar}{2}\left(\psi^{*} \frac{\partial \psi}{\partial t}-\text { c.c. }\right)=-\frac{i \hbar}{2}\left(\psi^{*} \nabla_{r} \psi-\text { c.c. }\right) \frac{d \mathbf{R}}{d t},
$$

where use is made of the relation:

$$
\frac{\partial \psi}{\partial t}=\frac{d \mathbf{R}}{d t} \nabla_{R} \psi
$$

together with the relation $\nabla_{R}=-\nabla_{r}$ (that is, the the nabla with respect to the vortex center coordinate tuns out to be the derivative with respect to field argument $\mathbf{r}$ ). Now we adopt the polar form of the order parameter

$$
\psi=\sqrt{\rho} \exp \left[i \frac{m}{\hbar} \alpha(\mathbf{r}-\mathbf{R}(t))\right] .
$$

Here $\rho=\rho(\mathbf{r}-\mathbf{R}(t))$ is the density, which vanishes at the vortex center $\mathbf{r}=\mathbf{R}$ and tends to constant value: $\rho=\rho_{0}$ outside the coherent length. The first term of $L$ (denoted by $L_{c}$ that is called the canonical term) is calculated as

$$
L_{c}=\int \mathbf{j} \cdot \frac{d \mathbf{R}}{d t} d \sigma \equiv \int\left(j_{x} \cdot \dot{X}+j_{y} \cdot \dot{Y}+j_{z} \cdot \dot{Z}\right) d \sigma
$$

where mass current $\mathbf{j}=m \rho \nabla \alpha=m \rho \mathbf{v}$ is defined. On the other hand, the Hamiltonian term becomes

$$
H=\int\left[\frac{1}{2} m \rho \mathbf{v}^{2}+\frac{1}{4 \rho}(\nabla \rho)^{2}+V(\rho)\right] d \sigma
$$

The first term means the fluid kinetic energy and the second and third terms are the internal energy, which is quoted as $U(\rho)$. The explicit form will be given later.

Now the equation of motion for the vortex center is derived from the Euler-Lagrange equation:

$$
\frac{d}{d t}\left(\frac{\partial L}{\partial \dot{\mathbf{R}}}\right)-\frac{\partial L}{\partial \mathbf{R}}=0
$$

The contribution from the Hamiltonian is

$$
\frac{\partial L}{\partial \mathbf{R}}=\frac{\partial}{\partial \mathbf{R}} \int \mathcal{H} d \sigma=\frac{\partial H}{\partial \mathbf{R}} .
$$

On the other hand, the canonical term is manipulated as follows: First by differentiating the under integral symbol, we have

$$
\frac{d}{d t}\left(\frac{\partial L_{c}}{\partial \dot{X}}\right)=\int \frac{d j_{x}}{d t} d \sigma
$$

together with $\frac{d j_{x}}{d t}=\frac{\partial j_{x}}{\partial X} \dot{X}+\frac{\partial j_{x}}{\partial Y} \dot{Y}+\frac{\partial j_{x}}{\partial Z} \dot{Z}$ and

$$
\frac{\partial L_{c}}{\partial X}=\int\left(\frac{\partial j_{x}}{\partial X} \dot{X}+\frac{\partial j_{y}}{\partial X} \dot{Y}+\frac{\partial j_{z}}{\partial X} \dot{Z}\right) d \sigma .
$$

Hence we obtain

$$
\frac{d}{d t}\left(\frac{\partial L_{c}}{\partial \dot{\mathbf{R}}}\right)-\frac{\partial L_{c}}{\partial \mathbf{R}}=\int\{(\nabla \times \mathbf{j}) \times \dot{\mathbf{R}}\} d \sigma,
$$

where use is made of $\frac{\partial \mathbf{j}}{\partial X}=-\frac{\partial \mathbf{j}}{\partial x}$. By evaluating the RHS of (8) (see Appendix A), we arrive at the Kirchhoff equation of motion for the vortex center

$$
\mu \Omega\left(\hat{\mathbf{R}} \times \frac{d \hat{\mathbf{R}}}{d t}\right)=\frac{\partial H}{\partial \hat{\mathbf{R}}}
$$

with $\Omega=m \rho_{0} a^{2}$ and replacing as $H \rightarrow H / a$. $\mu$ means the "vortex charge", which is introduced a multiplied factor in velocity field (Appendix A). Eq.(9) coincides with the equation of motion for classical vortex on sphere [8, 9], which is derived in the framework of classical fluid mechanics. This equation has a simple dynamical meaning; the balance of two types of forces; the left hand side represents the Magnus force and the right hand side is the gradient force coming from the Hamiltonian (that plays a role of potential energy in analogy with classical particle mechanics). This equation of motion can be written in an alternative form:

$$
\mu \Omega \frac{d \hat{\mathbf{R}}}{d t}=-\hat{\mathbf{R}} \times \frac{\partial H}{\partial \hat{\mathbf{R}}} .
$$

The equation of motion is rewritten in a form of Hamiltonian equation of motion. Namely, by using the component for spherical vector basis

$$
\frac{d \hat{\mathbf{R}}}{d t}=(0, \dot{\Theta}, \sin \Theta \dot{\Phi})
$$

the equation of motion is written in terms of the angular form:

$$
\mu \Omega \dot{\Theta}=\frac{1}{\sin \Theta} \frac{\partial H}{\partial \Phi}, \mu \Omega \dot{\Phi}=-\frac{1}{\sin \Theta} \frac{\partial H}{\partial \Theta} .
$$

This is of the same form as the equation of motion for spin (see, e.g. [15, 16]). The effective action corresponding to the equation of motion may be given as

$$
S=\int[\mu \Omega(1-\cos \Theta) \dot{\Phi}-H] d t .
$$

Quantization of vortex charge: We examine a special feature of the above form of action function; namely, the line integral. As a particular case, we consider the integral along a closed loop, say, $C$. This can be written by the surface surrounding $C$; where there is ambiguity choosing it,

$$
\begin{aligned}
\int_{C} \mu \Omega(1-\cos \Theta) d \Phi & =\mu \Omega \int_{S} \sin \Theta d \Theta d \Phi \\
& =-\mu \Omega \int_{\hat{S}} \sin \Theta d \Theta d \Phi
\end{aligned}
$$

which consists of the upper or lower surfaces, respectively, say $S$ and $\hat{S}$ that are complement each other; $S+\hat{S}=S^{2}$. This ambiguity is related to the gauge choice [17]. Upon quantization, the ambiguity is solved; that is expressed as an exponentiation;

$$
\exp \left[\frac{i}{\hbar} \mu \Omega \int_{S} \sin \Theta d \Theta d \Phi\right]=\exp \left[-\frac{i}{\hbar} \mu \Omega \int_{\hat{S}} \sin \Theta d \Theta d \Phi\right]
$$


then we obtain

$$
\mu \Omega \int_{S^{2}} \sin \Theta d \Theta \wedge d \Phi=2 n \pi \hbar \quad(\mathrm{n}=\text { integer })
$$

which leads to the Dirac monopole quantization:

$$
4 \pi \mu \Omega \equiv \mu M=\hbar \frac{n}{2},
$$

where $M=4 \pi \Omega$, which means the mass of fluid on the sphere. Equation (14) is just the same as the relation between electric and magnetic charge for the case of quantized monopole.

Hamiltonian term; pinning potential:

The Hamiltonian term is evaluated as follows: First we note that the fluid kinetic energy (7) together with the second term which is given by the gradient of the density profile. These two are independent of the position of vortex center for the case of single vortex. We need to settle a coordinate that is relative to the vortex center. Then the term satisfying this criterion is the last term $V(\rho)$. In order to express this explicitly, we consider the interaction energy with the pinning centers that is built in the superfluid, which is written in a form

$$
U=\int \psi^{*}(\mathbf{r}) \psi(\mathbf{r}) V(\mathbf{r}) d \mathbf{r}
$$

and noting that the density profile is given as $|\psi(\mathbf{r})|^{2}=$ $\rho_{0}+\tilde{\rho}(|\mathbf{r}-\mathbf{R}|)$ where $\rho_{0}$ is the uniform background term and $\tilde{\rho}$ describes the vortex profile, which may be given by a Gaussian form with the central peak at the vortex center. The interaction $V(\mathbf{r})$ can be chosen such that it couples in a contact form; namely, that may be given by the delta function:

$$
V(\mathbf{r})=V_{0} \delta(\mathbf{r}-\mathbf{a})
$$

with the pinning center a, then we have $U=V_{0} \tilde{\rho}(\mathbf{R}-$ a) up to an additional constant term coming from the uniform background. If we take $\mathbf{a}=(0,0, a)$ as the northpole: it turns out to be

$$
U=V_{0} \tilde{\rho}(a \sqrt{\mid(1-\cos \Theta \mid})
$$

where $\Theta$ denotes the angle between the vortex center and the north-pole. In the case that there are several pinning centers located at $\mathbf{a}_{k}, \mathrm{U}$ is given by

$$
U=\sum_{k=1}^{N} V_{0 k}\left(\tilde{\rho}\left(\left|\mathbf{R}-\mathbf{a}_{k}\right|\right)\right.
$$

\section{THE LANGEVIN AND FOKKER-PLANCK EQUATIONS}

In this section we consider the stochastic equation for the vortex motion by following the analogy between spin equation of motion.

\section{A. Spin analogy and the functional integral}

As is remarked in the previous section, the equation of motion (10) is of same form as the spin equation of motion; the vector $\hat{\mathbf{R}}$ plays a role for the spin vector, which we call the pseudo-spin. Here we introduce $\mathbf{J}=$ $J \hat{\mathbf{R}}$, and $J \equiv \mu \Omega$ corresponds to the spin magnitude. In terms of $\mathbf{J}$, the above equation is written in a scaled form:

$$
\frac{d \mathbf{J}}{d t}=-\mathbf{J} \times \frac{\partial H}{\partial \mathbf{J}} .
$$

The right hand side of the equation represents the torque, namely, $\frac{\partial H}{\partial \mathbf{J}}$ is nothing but the magnetic field acting on the pseudo-spin, which may be called "pseudo-magnetic field". From physical point of view, the analogy between spin and vortex is not surprising, since the vortex may be regarded as a region where the angular momentum of the fluid is concentrated.

Furthermore, following the well known fact in the spin theory, it can be extended to include the effect of dissipation: such that $\nabla H$ is replaced by $\nabla H+\eta \frac{d \mathbf{J}}{d t}$ in (18); with $\eta$, the dissipative coefficient. Having solved the equation for $\frac{d \mathbf{J}}{d t}$, it follows (which is known as "Landau-Lifschitz equation" [18]):

$$
\frac{d \mathbf{J}}{d t}+\mathbf{A}(\mathbf{J})=0
$$

where we use the notational convention:

$$
\mathbf{A}(\mathbf{J})=\frac{1}{1+\eta^{2} J^{2}}\left[\mathbf{J} \times \frac{\partial H}{\partial \mathbf{J}}-\eta J^{2} \frac{\partial H}{\partial \mathbf{J}}\right],
$$

We now treat the Brownian motion of point vortex that is caused by random effects of several origins; the temperature fluctuations, and inevitably existing impurities. Here we follow an analogy that is expected to occur between a single vortex and a ferromagnetic spin which can be observed in a single ferromagnetic domain [11]. For the case of ferromagnetic spin, the simplification is adopted; the random thermal fluctuations have a correlation time much shorter than the response time of the system. The response time of a single domain particle is of the same order as the reciprocal of the gyro-magnetic resonance frequency. However, the case of the vortex on a sphere, it is not feasible to estimate the ratio between two characteristic times, so we adopt it as a working hypothesis.

In what follows we consider that the fluctuation effect gives rise to the randomness of the pseudo-magnetic field; which may be written as $\mathbf{b}$, so we replace $\frac{\partial H}{\partial \mathbf{J}} \rightarrow \frac{\partial H}{\partial \mathbf{J}}+\mathbf{b}$. Here $\mathbf{b}(t)$ is assumed to be the Gaussian white noise as a random uncorrelated magnetic field. Hence we have

$$
\frac{d \mathbf{J}}{d t}+\mathbf{A}(\mathbf{J})=\mathbf{c}(t)
$$

where $\mathbf{c}(t)$ the right-hand side, that is

$$
\mathbf{c}(t)=\frac{1}{1+\eta^{2} J^{2}}\left(\mathbf{J} \times \mathbf{b}-\eta J^{2} \mathbf{b}\right)
$$


which is a combination of random force and torque. As a result of of the random uncorrelated features of the function $\mathbf{b}$, we assume that $\mathbf{c}$ can also be expected to be Gaussian white noise, which is expressed as

$$
\begin{aligned}
\left\langle c_{i}(t)\right\rangle & =0, \\
\left\langle c_{i}(t) c_{j}(t+u)\right\rangle & =h \delta_{i, j} \delta(u) .
\end{aligned}
$$

with $\delta(u)$ the delta function. It should be noted that the validity of the above form of white noise is not easy to justify and it is nothing else than the working hypothesis. The white noise is introduced to express that the random magnetic field is correlated on time-scales much smaller than the characteristic response time of the pseudo-spin system. It is assumed that $\left\langle\mathbf{c}^{2}\right\rangle=2 h$, and its probability distribution may be given by the standard Gaussian functional form [13, 14]:

$$
P[\mathbf{c}(t)]=\exp \left[-\frac{1}{2 h} \int_{0}^{t} \mathbf{c}^{2}(t) d t\right]
$$

Using this distribution, the propagator $K$ between two definite spin states at two different times, is given by the functional integral:

$$
\begin{aligned}
K[\mathbf{J}(t) \mid \mathbf{J}(0)] & =\int \prod_{t} \delta\left[\frac{d \mathbf{J}}{d t}+\mathbf{A}(\mathbf{J}(t))-\mathbf{c}(t)\right] \\
& \times \exp \left[-\int \frac{\mathbf{c}^{2}(t)}{2 h} d t\right] \mathcal{D}[\mathbf{J}] \mathcal{D}[\mathbf{c}(t)]
\end{aligned}
$$

with $\delta$ being the Dirac delta- functional. We need to calculate the Gaussian integral with respect to $\mathbf{c}(t)$, in which the functional Jacobian factor should be carried out. The details of this is briefly given in Appendix B and the result is

$$
K[\mathbf{J}(t) \mid \mathbf{J}(0)]=\int \exp \left[-\frac{1}{2 h} \int_{0}^{t}\left(\frac{d \mathbf{J}}{d t}+\mathbf{A}(\mathbf{J})\right)^{2} d t\right] \mathcal{D}[\mathbf{J}]
$$

Expanding the squared term inside the exponential term, one can put this functional integral in a familiar form of the path integral for a particle in the vector potential $\mathbf{A}$ and the scalar potential $V=\frac{\mathbf{A}^{2}}{2}$. The parameter $h$ just corresponds to the Planck constant. We can formally write the above functional integral by the quantum mechanical path integral, that is, by using the imaginary time $\tau=i t$

$$
K=\int \exp \left[\frac{i}{h} \int\left\{\frac{1}{2}\left(\frac{d \mathbf{J}}{d \tau}\right)^{2}+i \mathbf{A} \cdot \frac{d \mathbf{J}}{d \tau}-V\right\} d \tau\right] \mathcal{D} \mathbf{J}(t)
$$

\section{B. The Fokker-Planck equation}

The derivation of the Fokker-Planck is derived most directly by using the above path integral ([19]). If we introduce the "wave function" $\psi(\mathbf{J}, \tau)$, we have the integral equation:

$$
\Psi(\mathbf{J}, \tau)=\int K[\mathbf{J}(\tau) \mid \mathbf{J}(0)] \Psi(\mathbf{J}, 0) d \mathbf{J}(0)
$$

and following the standard procedure, we obtain the Schroedinger equation:

$$
i h \frac{\partial \Psi}{\partial \tau}=\left[\frac{1}{2}(\mathbf{P}+i \mathbf{A})^{2}+V\right] \Psi
$$

with $\mathbf{P}=-i h \frac{\partial}{\partial \mathbf{J}} \equiv-i h \nabla$. Returning to the real time; namely, $i \frac{\partial}{\partial \tau}=-\frac{\partial}{\partial t}$ : then writing $\Psi \rightarrow P$ : we arrive at the standard form of the FP equation [20]:

$$
\frac{\partial P}{\partial t}=h \nabla^{2} P-\nabla \cdot(\mathbf{A} P)
$$

This can be rewritten as the continuity equation that is written as $\frac{\partial P}{\partial t}+\nabla \cdot \mathbf{s}=0$, where $\mathbf{s}$ denotes the probability current $\mathbf{s}=-h \nabla P+\mathbf{A} P$ and the components in polar coordinate $(\Theta, \Phi)$ are

$$
\begin{aligned}
& s_{\theta}=-h \frac{\partial P}{\partial \Theta}+\frac{P}{1+\eta^{2} J^{2}}\left(\eta J^{2} \frac{\partial H}{\partial \Theta}-\frac{1}{\sin \Theta} \frac{\partial H}{\partial \Phi}\right) \\
& s_{\phi}=-\frac{h}{\sin \Theta} \frac{\partial P}{\partial \Phi}+\frac{P}{1+\eta^{2} J^{2}}\left(\frac{\partial H}{\partial \Theta}+\frac{\eta J^{2}}{\sin \Theta} \frac{\partial H}{\partial \Phi}\right)
\end{aligned}
$$

and the FP equation is written as

$$
\frac{\partial P}{\partial t}=-\frac{1}{\sin \Theta}\left\{\frac{\partial}{\partial \Theta}\left(\sin \theta s_{\theta}\right)+\frac{\partial s_{\phi}}{\partial \Phi}\right\}
$$

We here consider several general consequences from the FP equation.

(i): The stationary distribution: We consider $\frac{\partial P}{\partial t}=0$, and we put ansatz of the Boltzmann distribution: $P(\mathbf{J})=$ $\exp [-\beta H]$ with $\beta$ the inverse temperature $\beta=\frac{1}{k_{B} T}$. By substituting this into the RHS of the FP equation, we get

$$
\left(\beta h-\frac{\eta J^{2}}{1+\eta^{2} J^{2}}\right)\left\{\nabla^{2} H-\beta(\nabla H)^{2}\right\}=0
$$

From this it follows the relation:

$$
\beta h-\frac{\eta J^{2}}{1+\eta^{2} J^{2}}=0
$$

This relation is just the fluctuation dissipation relation, which establishes the relation between the dissipation coefficient $\eta$ and the diffusion (fluctuation) coefficient $h$.

(ii): Evolution equation for average value [22]: The $\mathrm{FP}$ equation enables us to evaluate the average of functions on the sphere; $F(\mathbf{J})$ : that is given by $\langle F\rangle=$ $\int F(\mathbf{J}) P(\mathbf{J}) d \mathbf{J}$ :

$$
\frac{\partial\langle F\rangle}{\partial t}=h\left\langle\nabla^{2} F\right\rangle-\langle\nabla F \cdot \mathbf{A}\rangle
$$

where use is made of partial integration. Here as an interesting example, we take up $F(\mathbf{J})=J_{i}^{2}(i=x, y, z)$. Further we choose $\mathbf{A}=\gamma \mathbf{J}$, which represents the relaxation effect, so we have for $\langle F\rangle$,

$$
\frac{d\left\langle J_{i}^{2}\right\rangle}{d t}=2 h-\gamma\left\langle J_{i}^{2}\right\rangle
$$


This is solved as

$$
\left\langle J_{i}^{2}\right\rangle=\frac{2 h}{\gamma}\left(1-\exp \left[-\frac{\gamma}{2 h} t\right]\right)
$$

Namely, this shows a typical relaxation behavior of $\left\langle J_{i}^{2}\right.$ leading to the asymptotic value $\left\langle J_{i}^{2}(\infty)\right\rangle=\frac{2 h}{\gamma}$.

\section{SIMPLE EXAMPLES: THE EFFECT OF PINNING POTENTIAL}

The FP equation is of peculiar form that is somewhat different from the usual one that consists of the potential term, so we need to invoke specific techniques to deal with this. We now consider an example that can be treated by analytic as well as approximate ways.

As the Hamiltonian we take the type given by the form such that it is given as a function of $\Theta$ : That is given by the pinning potential (17) which comes from the pinning center located at the north pole. As a concrete form we consider the case that the profile $\tilde{\rho}$ is given by the Gaussian [21] : $\tilde{\rho}=\exp \left[-\frac{(\mathbf{r}-\mathbf{R})^{2}}{\alpha}\right]$ with the vortex size $\alpha$, hence we have

$$
H(\Theta)=V_{0} \exp \left[-a^{2}(1-\cos \Theta) / \alpha\right] \equiv V_{0} \tilde{H} .
$$

As another form, it is possible to adopt the potential arising from two pining centers; one is located at the north and the other is at the south pole: and is written as

$H(\Theta)=V_{0}\left\{\exp \left[-\frac{a^{2}}{\alpha}(1-\cos \Theta)\right]+\exp \left[-\frac{a^{2}}{\alpha}(1+\cos \Theta)\right]\right\}$

In the following we consider the two cases without or with the effect of dissipation.

(i) The case that there is no dissipation: $\eta=0$ : We examine this case in the semiclassical way based on the classical equation of motion; the "Lagrangian" in the (25) is is

$$
\mathcal{L}=\frac{1}{2} J^{2}\left[\dot{\Theta}^{2}+\sin ^{2} \Theta\left(\dot{\Phi}+\frac{V_{0}}{J \sin \Theta} \frac{d \tilde{H}}{d \Theta}\right)^{2}\right]
$$

We here examine a special solution such that $\dot{\Theta}=0$, which leads to $\Theta=\Theta_{0}$. Then, the functional integral is

$$
K=\int \exp \left[-\frac{1}{2 h} \int_{0}^{t} \sin ^{2} \Theta_{0}(\dot{\Phi}+\kappa)^{2} d t\right] \mathcal{D}(\Phi)
$$

with

$$
\kappa \equiv\left[\frac{V_{0}}{J \sin \Theta} \frac{d \tilde{H}}{d \Theta}\right]_{\Theta=\Theta_{0}}
$$

This is equivalent to the path integral for a particle on a circle; the corresponding FP equation becomes

$$
h \frac{\partial P}{\partial t}=\frac{h^{2}}{\sin ^{2} \Theta_{0}} \frac{\partial^{2} P}{\partial \Phi^{2}}-h \kappa \frac{\partial P}{\partial \Phi}
$$

which is a modified form of the diffusion equation. We assume the solution to be a form

$$
P(\Phi, t)=\sum_{n=-\infty}^{\infty} \exp \left[-\lambda_{n} t\right] f_{n}(\Phi)
$$

Here $f_{n}(\Phi)$ satisfies the eigenvalue equation

$$
\frac{h}{\sin ^{2} \Theta_{0}} \frac{d^{2} f_{n}}{d \Phi^{2}}-\kappa \frac{d f_{n}}{d \Phi}=-\lambda_{n} f_{n}
$$

We put $f_{n}(\Phi)=\exp [i n \Phi]$ ( $n=$ integers $)$, and the eigenvalue is obtained as

$$
\lambda_{n}=\frac{h}{\sin ^{2} \Theta_{0}} n^{2}+i n \kappa
$$

By substituting this into the (38), we have

$$
P(\Phi)=\sum_{n=-\infty}^{\infty} \exp \left[-\left(\frac{h}{\sin ^{2} \Theta_{0}} n^{2}+i n \kappa\right) t\right] \exp [i n \Phi]
$$

which turns out to be the theta function [23]. The expectation values for $\langle\mathbf{J}\rangle=\int_{0}^{2 \pi} \mathbf{J} P(\Phi, t) d \Phi$ is calculated as

$$
\begin{aligned}
& \left\langle J_{x}\right\rangle=J \sin \Theta_{0} \exp \left[-\frac{h t}{\sin \Theta_{0}}\right] \cos \kappa t \\
& \left\langle J_{y}\right\rangle==J \sin \Theta_{0} \exp \left[-\frac{h t}{\sin \Theta_{0}}\right] \sin \kappa t \\
& \left\langle J_{z}\right\rangle=J \cos \Theta_{0}
\end{aligned}
$$

The damping behavior of $\left(J_{x}, J_{y}\right)$ shows is similar to the diffusive behavior of spin in magnetic systems [24].

(ii) The case that there is dissipation: $\eta \neq 0$.

For this case, we look for the distribution function as a function of $\Theta$ only $P \equiv P(\Theta)$, so the stationary FP equation is given by

$\frac{h}{\sin \Theta} \frac{d}{d \Theta}\left(\sin \Theta \frac{d P}{d \Theta}\right)-\frac{V_{0} \eta J^{2}}{1+\eta^{2} J^{2}} \frac{1}{\sin \Theta} \frac{d}{d \Theta}\left(\sin \Theta \frac{d \tilde{H}}{d \Theta} P\right)=-E P$

which is rewritten as

$$
\frac{1}{\sin \Theta} \frac{d}{d \Theta}\left(\sin \Theta \frac{d P}{d \Theta}\right)-\frac{\epsilon}{\sin \Theta} \frac{d}{d \Theta}\left(\sin \Theta \frac{d \tilde{H}}{d \Theta} P\right)=-\lambda P
$$

Here we put $\epsilon=\frac{V_{0} \eta J^{2}}{h\left(1+\eta^{2} J^{2}\right)}$ and $\lambda=\frac{E}{h}$. This can be regarded as an expansion parameter. Noting that $\epsilon \equiv$ $\frac{V_{0} \eta J^{2}}{h\left(1+\eta^{2} J^{2}\right)}$ turns out to be $\epsilon=V_{0} \beta$ as a consequence of the fluctuation-dissipation relation (30), we can treat the expansion scheme in terms of the expansion parameter $\epsilon$

(a): We first consider the perturbation procedure: this may be relevant for the case that $V_{0} \beta<<1$, which means the temperature is higher enough the interaction strength satisfies $V_{0}<<k_{B} T$. Hence expanding in terms of power series with respect to $\epsilon$ :

$$
\begin{aligned}
P(\Theta) & =P^{(0)}(\Theta)+P^{(1)}(\Theta)+\cdots \\
\lambda & =\lambda_{0}+\lambda_{1}+\cdots
\end{aligned}
$$


Substituting this into the above eigenvalue equation, we can determine each order by iteration procedure: the zero-th order

$$
\frac{h}{\sin \Theta} \frac{d}{d \Theta}\left(\sin \Theta \frac{d P^{(0)}}{d \Theta}\right)=-\lambda_{0} P^{(0)}
$$

and the first order:

$$
\begin{array}{r}
\frac{h}{\sin \Theta} \frac{d}{d \Theta}\left(\sin \Theta \frac{d P^{(1)}}{d \Theta}\right)-\frac{\epsilon}{\sin \Theta} \frac{d}{d \Theta}\left(\sin \Theta \frac{d \tilde{H}}{d \Theta}\right) P^{(0)} \\
=-\lambda_{1} P^{(0)}-\lambda_{0} P^{(1)} .
\end{array}
$$

The zeroth order gives the Legendre polynomial: $P^{0}=$ $P_{l}(\Theta)$ and the corresponding eigenvalue $\lambda_{0}=l(l+1)$ with $l=1 \cdots$. Let us choose a particular $l$, namely, $P^{0}=P_{l}(\Theta)$; then the first order term is calculated to

$\lambda_{1}=\epsilon \int P_{l}(\Theta) \frac{1}{\sin \Theta} \frac{d}{d \Theta}\left(\sin \Theta \frac{d \tilde{H}}{d \Theta}\right) P_{l}(\Theta) d \Omega / \int P_{l}^{2}(\Theta) d \Omega$

$P^{(1)}$ can be expanded in terms of $P_{k}(\Theta): P^{(1)}=$ $\sum_{k} C_{k} P_{k}(\Theta)$, then we obtain the coefficients $C_{k}$

$$
C_{k}=\frac{V_{k l}}{\lambda_{0}(l)-\lambda_{0}(k)}
$$

with the matrix element:

$$
V_{k l} \equiv \epsilon \int P_{k}(\Theta) \frac{1}{\sin \Theta} \frac{d}{d \Theta}\left(\sin \Theta \frac{d \tilde{H}}{d \Theta}\right) P_{l}(\Theta) d \Omega
$$

for $l \neq k$. Using this, he second order shift of the eigenvalue is calculated

$$
\lambda_{2}=\epsilon^{2} \sum_{k \neq l} \frac{V_{k l}^{2}}{\lambda_{0}(l)-\lambda_{0}(k)}
$$

(b): We next examine the case that $\epsilon \simeq 1$, namely, $V_{0} \simeq k_{B} T$. In this case one has to use non-perturbative procedure [11]. In order to carry out this, we rewrite the FP equation in terms of the variable $\xi=\cos \Theta$ :

$$
\frac{d}{d \xi}\left[\left(1-\xi^{2}\right)\left\{\frac{d P}{d \xi}+\epsilon \frac{d \tilde{H}}{d \xi} P\right\}\right]=-\lambda P
$$

and if noting the relation

$$
\exp [-\epsilon \tilde{H}] \frac{d}{d \xi} \exp [\epsilon \tilde{H}]=\frac{d}{d \xi}+\epsilon \frac{d \tilde{H}}{d \xi}
$$

we have

$$
\frac{d}{d \xi}\left\{\left(1-\xi^{2}\right) \exp [-\epsilon \tilde{H}] \frac{d}{d \xi}(\exp [\epsilon \tilde{H}] P)\right\}=-\lambda P
$$

This can be further reduced by putting $Q=\exp [\epsilon \tilde{H}] P$,

$$
\frac{d}{d \xi}\left\{\left(1-\xi^{2}\right) \exp [-\epsilon \tilde{H}] \frac{d Q}{d \xi}\right\}=-\lambda \exp [-\epsilon \tilde{H}] Q
$$

This equation can be translated to the variational problem; namely, we introduce the "action";

$$
I[Q]=\int_{-1}^{1}\left(1-\xi^{2}\right) \exp [-\epsilon \tilde{H}]\left(\frac{d Q}{d \xi}\right)^{2} d \xi
$$

and the constraint coming from the normalization;

$$
\int_{-1}^{1} \exp [-\epsilon \tilde{H}] Q^{2} d \xi \equiv N[Q]=1
$$

Taking the "Euler-Lagrange equation" $\delta(I[Q]-\lambda N[Q])=$ 0 , we recover the above eigenvalue equation with $\lambda$ is nothing but the Lagrangian multiplier. We see that there is a trivial solution $Q_{0}=$ constant with the zero eigenvalue $\lambda_{0}=0$. Starting from this as the lowest solution, we have an algorithm to get the subsequent solutions; $Q_{n}(n=0, \cdots)$ by noting the orthogonality condition;

$$
\int_{-1}^{1} \exp [-\epsilon \tilde{H}] Q Q_{n} d \xi=0
$$

As a concrete case, we exemplify how to construct the second lowest state by taking a trial function given by the quadratic function $Q_{1}(\xi)=A+B \xi+C \xi^{2}$. Substituting this into (50), we have the linear relation between $A, B, C$ and $C$ can be expressed by $A, B$. Next (49) gives the quadratic equation for $A, B$. Finally (48) gives another quadratic form for $A, B$. Then, the problem is reduced to the minimization of the (48) written in terms of the quadratic form under the constraint (49) written by the quadratic equation. Having solved these, we arrive at the solution for $Q_{1}$. This procedure may proceed to step by step and we can have a series of the stationary solution of the FP equations.

\section{EXTENSION TO NON-SPHERICAL VORTEX}

Here we discuss that the non-spherical vortices can be readily obtained as variants of the vortex on the sphere that has been given above; we are concerned with the vortex on 2-dimensional plane and the vortex on pseudosphere.

\section{A. The case of planer vortex}

This can be regarded as a limiting case of the spherical vortex. For the vortex center coordinate $\mathbf{R}=(X, Y)$, the equation of motion for $\mathbf{R}$ is obtained as a special case of the infinite radius; $a \rightarrow \infty$;

$$
m \rho_{0} \mu\left(\mathbf{k} \times \frac{d \mathbf{R}}{d t}\right)=\frac{\partial H}{\partial \mathbf{R}}
$$

where $\mathbf{k}$ is the unit vector perpendicular to $(x, y)$ plane. The Langevin equation with dissipation together with 
the white noise $\mathbf{c}$ is given by

$$
\frac{d \mathbf{R}}{d t}+\mathbf{B}=\mathbf{c}
$$

Following the same procedure as the case of spherical vortex, we obtain the FP equation:

$$
h \frac{\partial P}{\partial t}=\nabla^{2} P-\nabla \cdot(\mathbf{B} P)
$$

where $\nabla=\left(\frac{\partial}{\partial X}, \frac{\partial}{\partial Y}\right)$ is just the nabla in two dimensional plane. In the above, $\mathbf{B}$ is

$$
\mathbf{B} \equiv \frac{1}{\left(m \rho_{0} \mu\right)^{2}+\eta^{2}}\left(m \rho_{0} \mu \mathbf{k} \times \frac{\partial H}{\partial \mathbf{R}}+\eta \frac{\partial H}{\partial \mathbf{R}}\right)
$$

This is essentially same as that has been used in the case of superconductivity vortex [10]. More detailed and complicated treatment was given for the actual superconductors in connection with high $T_{c}$ superconductivity [25, 26]. Our treatment here provide a general way to clarify the stochastic behavior of planer vortex. The details of the argument will be given elsewhere.

\section{B. The case of vortex on pseudo-sphere}

Next we consider a point vortex on the pseudo-sphere (which is denoted by $P S^{2}$ ) which has the surface with constant negative curvature. The superfluid coated on such pseudo-sphere may be realized in actual conditions as a local part of the superfluid that is put on the surface of complicated shape and this part can be regarded as a pseudo-sphere.

The point of the surface is described by the equation $x^{2}+y^{2}-z^{2}=-a^{2}$, which is parametrically written as

$$
x=\sinh \theta \cos \phi, x=\sinh \theta \sin \phi, z=\cosh \theta
$$

Let $\hat{\mathbf{R}} \equiv \mathbf{R} / a=(\sinh \Theta \cos \Phi, \sinh \Theta \sin \Phi, \cosh \Theta)$ be the vortex center, which satisfies 27.

$$
\mu \Omega \frac{d \hat{\mathbf{R}}}{d t}=-\hat{\mathbf{R}} * \frac{\partial H}{\partial \hat{\mathbf{R}}}
$$

Here the scalar and vector product, denoted as $*$, are defined by

$$
\begin{aligned}
\mathbf{a} \cdot \mathbf{b} & =a_{1} b_{1}+a_{2} b_{2}-a_{3} b_{3} \\
\mathbf{a} * \mathbf{b} & =\left(a_{2} b_{3}-a_{3} b_{2}, a_{3} b_{1}-a_{2} b_{3},-\left(a_{1} b_{2}-a_{2} b_{1}\right)\right)
\end{aligned}
$$

and the triple product formula

$$
\mathbf{a} *(\mathbf{b} * \mathbf{c})=(\mathbf{a} \cdot \mathbf{b}) \mathbf{c}-(\mathbf{a} \cdot \mathbf{c}) \mathbf{b}
$$

Note that the 3-rd component of the scalar product has a minus sign compared with the spherical case. Furthermore noted is that for the case of vortex on $P S^{2}$, there is no counterpart of the topological quantization, because
$P S^{2}$ is trivial in a topological sense, namely, this is isomorphic to 2-dimensional flat Euclidean space. We introduce the pseudo spin; $\mathbf{K}=K \hat{\mathbf{R}}$ with $K \equiv \mu \Omega$. The norm of $\mathbf{K}$ is given by $\mathbf{K}^{2}=-K^{2} . K$ is not quantized, which is a consequence of the non-existence of topological invariant for $P S^{2}$. According to the same manner as the sphere vortex, the Langevin equation with the dissipation is obtained as

$$
\begin{aligned}
\frac{d \mathbf{K}}{d t} & =\tilde{A}(\mathbf{K})+\tilde{\mathbf{c}} \\
\tilde{A}(\mathbf{K}) & \equiv \frac{1}{1-\eta^{2} K^{2}}\left(-\mathbf{K} * \frac{\partial H}{\partial \mathbf{K}}-\eta K^{2} \frac{\partial H}{\partial \mathbf{K}}\right)
\end{aligned}
$$

Here note that $c$, which is the random force, has the norm such that $\tilde{\mathbf{c}}^{2}=c_{1}^{2}+c_{2}^{2}-c_{3}^{2}$, which is a consequence of the non-Euclidean metric on the pseudo-sphere. By taking account of this fact and following the same procedure as the spherical vortex case, we arrive at the FP equation;

$$
\frac{\partial P}{\partial t}=-\frac{1}{\sinh \Theta}\left\{\frac{\partial}{\partial \Theta}\left(\sinh \theta s_{\theta}\right)+\frac{\partial s_{\phi}}{\partial \Phi}\right\}
$$

where the current is

$$
\begin{aligned}
& s_{\theta}=-h \frac{\partial P}{\partial \Theta}-\frac{P}{1-\eta^{2} K^{2}}\left(\eta K^{2} \frac{\partial H}{\partial \Theta}-\frac{1}{\sinh \Theta} \frac{\partial H}{\partial \Phi}\right) \\
& s_{\phi}=-\frac{h}{\sinh \Theta} \frac{\partial P}{\partial \Phi}+\frac{P}{1-\eta^{2} K^{2}}\left(\frac{\partial H}{\partial \Theta}-\frac{\eta K^{2}}{\sinh \Theta} \frac{\partial H}{\partial \Phi}\right)
\end{aligned}
$$

We can develop the approximate procedure in the same way as the spherical vortex. This may be realized by finding the pinning potential, which can be obtained by replacing $\cos \Theta \rightarrow \cosh \Theta$ (17). For developing the perturbation, the zeroth order (unperturbed) distribution function satisfies $\Theta$,

$$
\frac{1}{\sinh \Theta} \frac{\partial}{\partial \Theta}\left(\sinh \Theta \frac{\partial P}{\partial \Theta}\right)=\lambda P
$$

and the eigenvalue is given by $\lambda=\rho(\rho+1)$ with $\rho$ is the real number and the eigenfunction is written in terms of the integral representation [28]:

$$
P_{\rho}(\cosh \Theta)=\frac{1}{2 \pi} \int_{0}^{2 \pi}(\cosh \Theta+\sinh \Theta \cos \xi)^{\rho} d \xi
$$

Further, for the non-perturbation case, we can construct the similar equation with (47) as well as the (48), which is simply obtained by replacing $\xi=\cos \Theta$ by $\xi=\cosh \Theta$. Then we can apply the variation principle for $I[Q]$ leading to the approximation solutions for the FP equation.

\section{SUMMARY}

We have presented a general formalism of the stochastic theory of random behavior of the quantum vortex that is coated on the two-dimensional sphere. The randomness are caused from several origins. We have mind of 
the conventional assumption; temperature fluctuations. For the case of mesoscopic size we have in mind, the fluctuation of temperature is more appreciable than the macroscopic system from the relative sense. As the other origin, we can take into account of the one that is intrinsically built in the spherical geometry, for which we expect to have the irregular deviation from the ideal sphere in actual situation [29, 30].

To formulate the random behavior, we follow the analogy with the spherical vortex and spin. This analogy seems natural; since the vortex carries angular momentum of the fluid in a form of "lump" which is the small region that angular momentum is concentrated. From the technical point of view of stochastic theory, the present approach is closely connected with the magnetic system [11]. Having noted the analogy with spin, we can write the Langevin equation for the Brownian motion of vortex, which can be converted to the Fokker-Planck equation using the functional integral method 13 , 14]. Though this present approach shares its idea with the FP equation for magnetic system[11], the present functional integral provides with the more direct means to access to the FP equation, that is quite different from the stochastic integral method (Ito integral) used in [11]. As a particular case, the approximate scheme has been developed for the case that the vortex Hamiltonian is given by the pinning potential. This case may be significant for practical analyses, since the pinning plays a crucial role for the vortex motion.

Finally, we gave an extension to the case of the variants of the spherical vortex; the planer vortex and the vortex on the pseudo-sphere. The vortex on the pseudo-sphere may be regarded as a step towards the quantum vortex that is defined on the more complicated two-dimensional manifold.

\section{Acknowledgement}

The author would like to thank professor R.Botet at Laboratoire de Physique Solides, Orsay for useful discussions on the Fokker-Planck equation.

\section{Appendix A: Calculation of $\nabla \times \mathbf{j}$}

We rewrite the surface integral of the right hand side of (18) by using the Stokes theorem:

$$
\int_{S}(\nabla \times \mathbf{j}) d \sigma=\int_{C} m \rho \mathbf{v} d \mathbf{s}
$$

with $C$ being boundary of $\mathrm{S}$. By noting the fact that if $C$ is chosen such that it is sufficiently remote from the vortex center, $\rho \rightarrow \rho_{0}$, we have

$$
\int_{C} m \rho \mathbf{v} d \mathbf{s}=m \rho_{0} \int_{C} \mathbf{v} \cdot d \mathbf{s} \equiv m \rho_{0} \int_{S} \omega d \sigma
$$

with the vorticity $\omega=\nabla \times \mathbf{v}$.

Next we give an explicit form for the velocity field, which is given by

$$
\mathbf{v}=\mu(\hat{r} \times \nabla f),
$$

where the vortex charge $\mu$ is introduced and $f$ is

$$
f=\log |\mathbf{r}-\mathbf{R}(t)|=\log a+\log |\hat{\mathbf{r}}-\hat{\mathbf{R}}(t)| .
$$

Here $\hat{\mathbf{r}}=\mathbf{r} / a, \hat{\mathbf{R}}=\mathbf{R} / a$, that is, the vector on unit sphere:

$$
\hat{R}=(\sin \Theta \cos \Phi, \sin \Theta \sin \Phi, \cos \Theta)
$$

At this point we adopt the polar coordinate system: $\hat{\mathbf{r}}=(\sin \theta \cos \phi, \sin \theta \sin \phi, \cos \theta)$ and the velocity field becomes

$$
\mathbf{v}=\mu\left(0,-\frac{1}{\sin \theta} \frac{\partial f}{\partial \phi}, \frac{\partial f}{\partial \theta}\right)
$$

Hence the vorticity is given by $\omega=\nabla \times \mathbf{v}=\left(\nabla^{2} f\right) \hat{r}$ with the Laplacian on 2-sphere

$$
\nabla^{2} f=\frac{1}{\sin \theta} \frac{\partial}{\partial \theta}\left(\sin \theta \frac{\partial f}{\partial \theta}\right)+\frac{1}{\sin ^{2} \theta} \frac{\partial^{2} f}{\partial \phi^{2}}=\delta(\hat{\mathbf{r}}-\hat{\mathbf{R}}(t)) .
$$

where we note $\nabla^{2} \log |\hat{\mathbf{r}}-\hat{\mathbf{R}}(t)|=\delta(\hat{\mathbf{r}}-\hat{\mathbf{R}}(t))$. From the above expression the vorticity for the spherical vortex directs to the normal of sphere. By substituting this into the Lagrangian equation of motion, we arrive at the Kirchhoff equation.

Vortex equation on a pseudo-sphere

Here we give a sketch for the vortex on pseudo-sphere $P S^{2}$

The procedure of calculation is completely same as the case of $S^{2}$; we need only the following changes:

$$
\nabla f=\left(0, \frac{\partial f}{\partial \theta}, \frac{1}{\sinh \theta} \frac{\partial f}{\partial \phi}\right) \equiv \frac{\partial f}{\partial \theta} \hat{\theta}+\frac{1}{\sinh \theta} \frac{\partial f}{\partial \phi} \hat{\phi}
$$

where $\hat{\theta}, \hat{\phi}$ means the unit vector in the direction of $(\theta, \phi)$. Noting that the velocity field is given by replacing $\sin \theta$ by $\sinh \theta$ and hence the vortex becomes $\omega=\nabla \times \mathbf{v}=\left(\nabla^{2} f\right) \hat{r}$ with the Laplacian on pseudo-sphere

$$
\nabla^{2} f=\frac{1}{\sinh \theta} \frac{\partial}{\partial}\left(\left(\sinh \theta \frac{\partial f}{\partial \theta}\right)+\frac{1}{\sinh ^{2} \theta} \frac{\partial^{2} f}{\partial \phi^{2}}\right.
$$

Hence, we obtain the equation of motion in the same form as the spherical case by noting the difference of vector product.

\section{Appendix B: Reduction of functional integral}

Here we show how to reduce the Gaussian functional integral. We introduce $\mathbf{G}=\frac{d \mathbf{J}}{d t}+\mathbf{A}(\mathbf{J})$. First we use the functional identity:

$$
\operatorname{det}\left[\frac{\delta \mathbf{G}(t)}{\delta \mathbf{J}(t)}\right]_{G=c} \int \prod_{t} \delta[\mathbf{G}(\mathbf{J})-\mathbf{c}] D[\mathbf{J}(t)]=1
$$


then we have the functional integral including the the Jacobian factor [31, 32]:

$$
\begin{aligned}
K & \left.=\int \operatorname{det}\left[\frac{\delta \mathbf{G}(t)}{\delta \mathbf{J}(t)}\right]_{\mathbf{G}=\mathbf{c}}\right] \int \prod_{t} \delta[\mathbf{G}(t)-\mathbf{c}(t)] D[\mathbf{J}(t)] \\
& \times \exp \left[-\frac{D}{2} \int \mathbf{c}^{2}(t) d t\right] D[\mathbf{c}(t)]
\end{aligned}
$$

Here we can remove the constraint that attaches to the det, namely, $\mathbf{G}=\mathbf{c}$. Because, it can be obtained by the well known the relation for the delta function $c(x) \delta(x-a)=c(a) \delta(x-a)$ and the determinant factor is generally regarded as a functional of $\mathbf{J}(t)$, so it does not depends on $\mathbf{c}(t)$ any more. Further, using an integral identity $\delta[g(x)]=\int \exp [i \lambda g(x)] d \lambda$, the above is written as a functional integration over three variables $\mathbf{J}, \mathbf{c}, \lambda$ : Thus we have

$$
\begin{aligned}
K & =\int \exp \left[i \int \lambda(t) \cdot\{\mathbf{G}(t)-\mathbf{c}(t)\} d t\right] \\
& \times \exp \left[-\frac{D}{2} \int \mathbf{c}^{2}(t) d t\right] D[\lambda(t)] \\
& \times \operatorname{det}\left[\frac{\delta \mathbf{G}(t)}{\delta \mathbf{J}(t)}\right] D[\mathbf{c}(t)] D[\mathbf{J}(t)]
\end{aligned}
$$

By accomplishing the two Gaussian functional integrals for $\mathbf{c}$ and $\lambda(t)$ successively, we obtain (25).
[1] H.Lamb, Hydrodynamics Cambridge University Press, Cambridge, 1935.

[2] L.Onsager, Nouvo Cimento 69, 279(1949) .

[3] A.L.Fetter, Phys.Rev.162,143(1967).

[4] R.Y.Chiao, A.Hansen, and A.A. Moulthrop, Phys.Lett.Lett. 54,1339(1985).

[5] M.Rasetti , and T.Regge, Physica 80A,217(1975).

[6] D.Mermin and T.L.Ho, Phys. Rev.Lett. 36(1976)594.

[7] T W B Kibble, J. Phys. A9,1387(1976) .

[8] Y.Kimura and H.Okamoto, J.Phys.Soc.Jpn. 56,4203(1987).

[9] R.Kidambi and P.K.Newton, Physica D140,95(2000).

[10] A.T.Dorsey, Phys.Rev. B46,)8376(1992.

[11] W.F.Brown, Phys.Rev.130,1677(1963).

[12] H.Kuratsuji, Phys.Rev.Lett. 68,1746(1992).

[13] M.Fibich and E.Helfand, Phys.Rev.183,265(1969).

[14] J.Zittarz and J.S. Langer, Phys.Rev.148,741(1966).

[15] H.Kuratsuji and T.Suzuki, J.Math.Phys.21,472(1980).

[16] H.Kuratsuji and Y.Mizobuchi, Phys.Lett.82A, 279(1981).

[17] J.J.Sakurai, Modern Quantum Mechanics, second edition, Addision Wesley, New York, 1995.

[18] L.Landau and E.Lifschitz, Phys.Z.Sovietunion 8,153 (1935).

[19] R.Feynman and A.R.Hibbs, Quantum Mechanics and Path Integral, Macgraw-Hill, New York, 1965.
[20] R.Kubo, M.Toda and N.Hashitsume, Statistical Physics II, 2-nd edition, Springer Verlag, New York, 1985.

[21] Alternatively we can choose $H=V_{0}|1-\cos \Theta|$ (for $\mid 1-$ $\cos \Theta \mid<\alpha$ ) and $H=0$ for otherwise.

[22] F.Dyson, J.Math.Phys.3, 1191 (1962).

[23] M.Toda, Introduction to elliptic functions(in Japanese), Nihon Hyoronsha, Tokyo, 2004.

[24] K.Yoshida, Magnetism (in Japanese),Iwanami Shoten, Tokyo, 2008.

[25] M.V.Feigelman and V.M.Vinokur, Phys.Rev.B41, 896(1990).

[26] Y.Enomoto, Phys.Lett.A161, 185 (1991).

[27] This equation of motion is similar to the one for the equation of motion of pseudo-spin on the non-compact phase space; $S U(1,1) / U(1)$ : See, M.Matsumoto and H.Kuratsui, Phys. Lett. A195, 18(1994).

[28] S.Helgason, Differential geometry and symmetric space, Academic press, New York, 1962.

[29] A.M.Turner, V.Vitelli and D.R.Nelson, Rev. Mod. Phys. $82,1301(2010)$

[30] M.J.Bowick and L.Gioni, Adv.Phys.58,449 (2009) and references therein.

[31] L.Faddeev and V.Popov, Phys.Lett.25B, 29 (1967).

[32] J.Zinn-Justin, Quantum field theory and critical phenomena, Oxford University press, Oxford, 1990. 